\title{
Effect of Nd:YAG Laser Capsulotomy on Anterior Segment Parameters in Patients with Posterior Capsular Opacification after Phacovitrectomy
}

\author{
Min Ho Shin ${ }^{1,2}$, Hyun Ji Kang ${ }^{1,2}$, Seong Jae Kim ${ }^{1-3}$, In Young Chung ${ }^{1-3}$, Seong Wook Seo ${ }^{1-3}$, Ji Myong Yoo ${ }^{1-3}$, \\ Jong Moon Park ${ }^{1,3,4}$, Yong Seop Han ${ }^{1,3,4}$

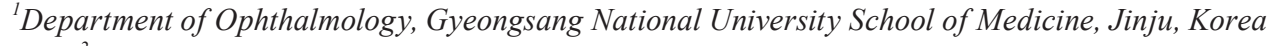 \\ ${ }^{2}$ Department of Ophthalmology, Gyeongsang National University Hospital, Jinju, Korea \\ ${ }^{3}$ Institute of Health Sciences, Gyeongsang National University School of Medicine, Jinju, Korea \\ ${ }^{4}$ Department of Ophthalmology, Gyeongsang National University Changwon Hospital, Changwon, Korea
}

Purpose: To compare changes in anterior segment parameters after Nd:YAG laser capsulotomy in eyes that underwent either combined phacovitrectomy or cataract surgery.

Methods: This retrospective study enrolled 37 eyes of 35 patients with posterior capsular opacification treated with combined phacovitrectomy (group A), and 35 eyes of 32 patients with posterior capsular opacification treated with cataract surgery (group B). Anterior segment parameters, including anterior chamber depth (ACD), anterior chamber angle, and anterior chamber volume, were measured by a Pentacam before Nd:YAG laser capsulotomy and 1 hour, 1 day, 1 week, 1 month, and 3 months after this treatment.

Results: In the cataract surgery group, the ACD was significantly lower 1 day $(3.75 \pm 0.74 \mathrm{~mm}), 1$ week $(3.73 \pm 0.24$ $\mathrm{mm})$, and 3 months $(3.74 \pm 0.33 \mathrm{~mm})$ after Nd:YAG laser capsulotomy compared with the pretreatment value $(4.20 \pm 0.62 \mathrm{~mm}, p=0.002)$. By contrast, the ACD did not change significantly over time in the combined phacovitrectomy group. The ACD differed significantly between the two groups at 1 week, 1 month, and 3 months after capsulotomy. There were no significant changes in the anterior chamber volume, anterior chamber angle, central corneal thickness, or pupil size from before to after capsulotomy in either group. A non-significant trend toward myopic shift was observed in group $\mathrm{A}(p=0.072)$ and $\mathrm{B}(p=0.055)$.

Conclusions: The results of the present study may help determine the power of the intraocular lens in patients who underwent combined surgery or cataract surgery and who will receive Nd:YAG laser capsulotomy.

Key Words: Anterior chamber parameters, Nd:YAG laser posterior capsulotomy, Phacovitrectomy, Posterior capsular opacification

Received: February 20, 2018 Accepted: April 22, 2018

Corresponding Author: Yong Seop Han, MD, PhD. Department of Ophthalmology, Gyeongsang National University Changwon Hospital, \#11 Samjeongja-ro, Seongsan-gu, Changwon 51472, Korea. Tel: 82-55-7508864, Fax: 82-55-758-4158, E-mail: medcabin@naver.com
Posterior capsular opacification (PCO) is the most common complication of cataract surgery and is characterized by monocular diplopia, light scattering, and reductions in visual acuity and contrast sensitivity [1,2]. Although the incidence of PCO varies among studies, rates as high as 
$11.8 \%$ at 1 year after cataract surgery, $20.7 \%$ at 3 years, and $28.4 \%$ at 5 years have been reported [3]. Whereas peripheral or mild PCO does not have a significant impact on visual acuity, PCO occurring within $3 \mathrm{~mm}$ of the central posterior capsule affects visual acuity significantly [4].

Since its introduction in 1980, Nd:YAG laser capsulotomy has become a standard treatment to improve visual acuity in pseudophakic outpatients with PCO [5,6]. It is important to evaluate anterior and posterior chamber parameters before and after Nd:YAG laser capsulotomy because this treatment can cause complications, such as elevation of intraocular pressure (IOP), corneal injury, iris hemorrhage, uveitis, macular hole, anterior hyaloid surface injury, cystoid macular edema, retinal detachment, intraocular lens (IOL) injury, IOL dislocation, and changes in the refractive index [2,6-8]. Studies assessing the effects of Nd:YAG laser capsulotomy on anterior chamber parameters, including anterior chamber depth (ACD), anterior chamber angle (ACA), and the refractive index, have yielded conflicting results [7,9-13].

To our knowledge, data are lacking regarding the effects of Nd:YAG laser capsulotomy on anterior chamber parameters in patients that developed PCO after combined phacovitrectomy. This study was therefore designed to quantitatively evaluate anterior chamber parameters, including the $\mathrm{ACD}$, anterior chamber volume (ACV), and ACA, using a Pentacam before and after Nd:YAG laser capsulotomy in eyes that were treated with either combined phacovitrectomy or cataract surgery alone. In addition, the effects of Nd:YAG laser capsulotomy on visual acuity, refraction, and IOP were evaluated in both groups.

\section{Materials and Methods}

\section{Subjects}

The study was performed in accordance with the tenets of the Declaration of Helsinki and all federal laws. Informed consent was obtained from all patients, and the study was approved by the institutional review board of Gyeongsang National University Hospital (2016-06-019). This retrospective study involved patients with PCO affecting visual acuity who visited Gyeongsang National University Hospital from July 2014 to June 2016. Patients followed up for more than 3 months after capsulotomy were enrolled. Patients with a history of trauma, uveitis, glaucoma, or other sub- stantial ophthalmic diseases were excluded. Also excluded were patients who had undergone intraocular surgery within 6 months, patients who had undergone sulcus or scleral fixation of IOLs, and patients who had undergone pars plana vitrectomy using intraocular tamponades with silicone oil, which could affect refractive power.

The patients were divided into two groups: group A consisted of 37 eyes of 35 patients that had undergone combined cataract surgery (phacoemulsification with IOL implantation) and pars plana vitrectomy (phacovitrectomy); group B consisted of 35 eyes of 32 patients that had undergone cataract surgery (phacoemulsification with IOL implantation) alone. Cataract surgery was performed by making a superior clear corneal incision, followed by phacoemulsification and insertion of a one-piece hydrophobic acrylic IOL (Acrysof IQ; Alcon, Fort Worth, TX, USA) into the capsular bag. The incision site was not sutured. Vitrectomy consisted of a standard three-port pars plana vitrectomy with a palliative 23-gauge system.

\section{Nd:YAG laser capsulotomy and Pentacam measurement}

PCO was diagnosed by retroillumination with a slit lamp biomicroscope. All Nd:YAG laser capsulotomies were performed by the same surgeon in a single session, with a circular pattern (average diameter, 4.0 to $4.5 \mathrm{~mm}$ ). The energy level used in all eyes was $2.2 \mathrm{~mJ}$. The ACD, ACV, ACA, pupil size, and central corneal thickness (CCT) were measured by Pentacam (Oculus Optikgerate GmbH, Wetzlar, Germany) Scheimpflug imaging. All Pentacam measurements were obtained under standard dim light conditions in non-dilated eyes. The best-corrected visual acuity (BCVA), IOP, and refractive changes through cycloplegic refraction were measured before treatment and 1 hour, 1 day, 1 week, 1 month, and 3 months after Nd:YAG laser capsulotomy. All ocular examinations were performed by a single practitioner.

\section{Statistical analysis}

Statistical analysis was performed with SPSS ver. 14.0 (SPSS Inc., Chicago, IL, USA). Friedman tests were used to analyze changes in visual acuity, refractive power, and anterior chamber parameters after Nd:YAG laser capsulotomy. Paired $t$-tests were used to compare parameters measured before laser treatment with those measured 1 hour, 1 day, 1 week, 1 month, and 3 months after Nd:YAG laser 
capsulotomy. Mann-Whitney $U$-tests were used to compare the two groups at each time point. A $p$-value less than 0.05 was defined as statistically significant.

\section{Results}

\section{Baseline characteristics}

The present study evaluated 72 eyes of 67 patients, including 37 eyes of 35 patients that underwent combined phacovitrectomy for the treatment of retinal disease and cataract (group A), and 35 eyes of 32 patients that underwent cataract surgery alone (group B). The demographics and clinical characteristics of the patients are presented in Table 1. In group A, the preoperative diagnosis for vitrectomy was epiretinal membrane in 16 eyes, vitreous hemorrhage in 14 eyes, and vitreous opacity in seven eyes. Before capsulotomy, the mean BCVA was $0.46 \pm 0.33$ logarithm of the minimum angle of resolution in group A and $0.48 \pm 0.32$ logarithm of the minimum angle of resolution in group B, and the mean IOP was $15.3 \pm 2.1 \mathrm{mmHg}$ in group A and $15.9 \pm 1.5 \mathrm{mmHg}$ in group B. The two groups did not differ significantly in age, sex, laterality of the operated eye, or BCVA and IOP before capsulotomy.

\section{Anterior segment parameters}

The mean ACD before Nd:YAG laser capsulotomy was similar in groups A $(4.32 \pm 0.54 \mathrm{~mm})$ and B $(4.20 \pm 0.62$ $\mathrm{mm})$. Although the ACD was not altered significantly in group A, significant reductions in the ACD were observed 1 day $(3.75 \pm 0.74 \mathrm{~mm}), 1$ week $(3.73 \pm 0.24 \mathrm{~mm})$, and 3 months $(3.74 \pm 0.33 \mathrm{~mm})$ after Nd:YAG laser capsulotomy in group B $(p=0.003)$. Moreover, the ACD differed significantly between the two groups 1 week, 1 month, and 3 months after capsulotomy (Fig. 1). Nd:YAG laser capsulotomy did not significantly alter the ACV, ACA, CCT, or pupil size in either group, and there were no significant differences in these variables between the two groups (Table 2).

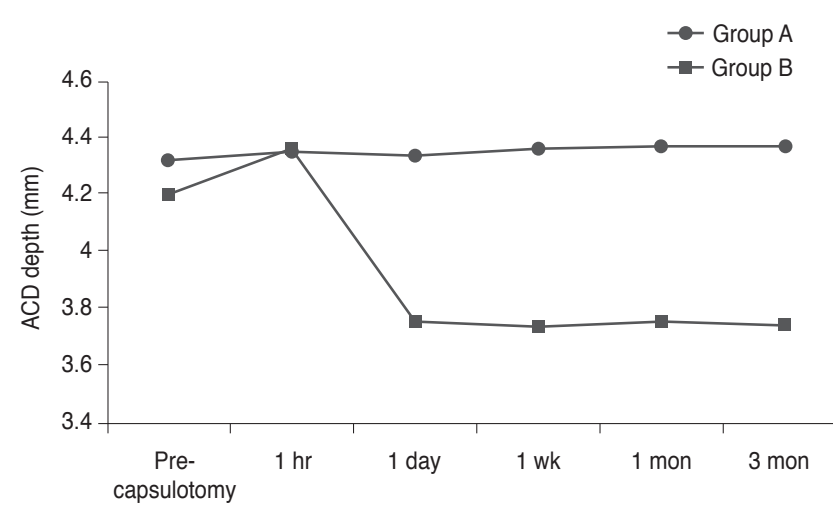

Fig. 1. Change in anterior chamber depth (ACD) after Nd:YAG laser capsulotomy. Group A: combined cataract operation (phacoemulsification with intraocular lens implantation) and vitrectomy. Group B: cataract operation (phacoemulsification with intraocular lens implantation).

Table 1. Patient demographics and clinical characteristics at baseline

\begin{tabular}{|c|c|c|c|c|}
\hline & Total patients & Group A & Group B & $p$-value ${ }^{*}$ \\
\hline No. of eyes (patients) & $72(67)$ & $37(35)$ & $35(32)$ & \\
\hline \multicolumn{5}{|l|}{ Sex } \\
\hline Male & 32 & 17 & 15 & 0.832 \\
\hline Female & 40 & 20 & 20 & 0.856 \\
\hline Age (yr) & $55.5 \pm 11.2$ & $57.6 \pm 11.5$ & $52.9 \pm 10.2$ & 0.786 \\
\hline \multicolumn{5}{|l|}{ Laterality } \\
\hline Right eye & 38 & 20 & 18 & 0.845 \\
\hline Left eye & 34 & 17 & 17 & 0.884 \\
\hline Preoperative BCVA (logMAR) & $0.47 \pm 0.32$ & $0.46 \pm 0.33$ & $0.48 \pm 0.32$ & 0.832 \\
\hline Basal IOP (mmHg) & $15.6 \pm 2.2$ & $15.3 \pm 2.1$ & $15.9 \pm 1.5$ & 0.708 \\
\hline
\end{tabular}

Values are presented as number or mean \pm standard deviation; Group A: combined cataract operation (phacoemulsification with intraocular lens implantation) and vitrectomy; Group B: cataract operation (phacoemulsification with intraocular lens implantation). $\mathrm{BCVA}=$ best-corrected visual acuity; $\log \mathrm{MAR}=$ logarithm of the minimum angle of resolution; IOP $=$ intraocular pressure. "Mann-Whitney $U$-test for comparisons between groups A and B. 
Table 2. Changes in anterior segment parameters after Nd:YAG laser capsulotomy

\begin{tabular}{|c|c|c|c|c|c|c|c|c|}
\hline & & Pre-capsulotomy & 1 Hour & 1 Day & 1 Week & 1 Month & 3 Months & $p$-value ${ }^{*}$ \\
\hline \multirow{5}{*}{$\begin{array}{l}\mathrm{ACD} \\
(\mathrm{mm})\end{array}$} & Group A & $4.32 \pm 0.54$ & $4.35 \pm 0.66$ & $4.34 \pm 0.52$ & $4.36 \pm 0.42$ & $4.37 \pm 0.36$ & $4.37 \pm 0.31$ & 0.245 \\
\hline & $p$-value ${ }^{\dagger}$ & - & 0.732 & 0.774 & 0.523 & 0.233 & 0.233 & - \\
\hline & Group B & $4.20 \pm 0.62$ & $4.36 \pm 0.88$ & $3.75 \pm 0.74$ & $3.73 \pm 0.24$ & $3.75 \pm 0.26$ & $3.74 \pm 0.33$ & 0.003 \\
\hline & $p$-value & - & 0.245 & 0.015 & 0.013 & 0.015 & 0.015 & - \\
\hline & $p$-value ${ }^{\S}$ & 0.570 & 0.413 & 0.089 & 0.004 & 0.004 & 0.003 & - \\
\hline \multirow{5}{*}{$\begin{array}{l}\mathrm{ACV} \\
(\mathrm{mL})\end{array}$} & Group A & $160.1 \pm 29.42$ & $162.8 \pm 29.35$ & $162.6 \pm 31.71$ & $161.8 \pm 21.44$ & $161.8 \pm 24.52$ & $162.1 \pm 24.71$ & 0.233 \\
\hline & $p$-value ${ }^{\dagger}$ & - & 0.724 & 0.769 & 0.829 & 0.829 & 0.788 & - \\
\hline & Group B & $159.8 \pm 24.80$ & $153.8 \pm 35.26$ & $143.3 \pm 30.45$ & $137.0 \pm 24.71$ & $138.2 \pm 26.21$ & $138.9 \pm 24.30$ & 0.13 \\
\hline & $p$-value & - & 0.228 & 0.127 & 0.055 & 0.062 & 0.074 & - \\
\hline & $p$-value ${ }^{\S}$ & 0.488 & 0.348 & 0.218 & 0.124 & 0.138 & 0.102 & - \\
\hline \multirow{5}{*}{$\begin{array}{c}\text { ACA } \\
\text { (degree) }\end{array}$} & Group A & $43.4 \pm 6.57$ & $43.5 \pm 5.99$ & $43.6 \pm 5.91$ & $43.6 \pm 5.94$ & $43.8 \pm 6.11$ & $44.4 \pm 5.77$ & 0.246 \\
\hline & $p$-value ${ }^{\dagger}$ & - & 0.823 & 0.775 & 0.780 & 0.438 & 0.247 & - \\
\hline & Group B & $42.2 \pm 5.96$ & $42.6 \pm 5.74$ & $43.2 \pm 6.48$ & $45.4 \pm 6.92$ & $44.5 \pm 6.78$ & $44.2 \pm 7.13$ & 0.271 \\
\hline & $p$-value ${ }^{\ddagger}$ & - & 0.432 & 0.232 & 0.073 & 0.125 & 0.138 & - \\
\hline & $p$-value ${ }^{\S}$ & 0.482 & 0.658 & 0.792 & 0.653 & 0.413 & 0.749 & - \\
\hline \multirow{5}{*}{$\begin{array}{l}\text { CCT } \\
(\mu \mathrm{m})\end{array}$} & Group A & $548.5 \pm 41.8$ & $550.2 \pm 38.4$ & $542.1 \pm 20.4$ & $548.2 \pm 32.4$ & $550.8 \pm 34.7$ & $550.2 \pm 38.4$ & 0.643 \\
\hline & $p$-value ${ }^{\dagger}$ & - & 0.879 & 0.779 & 0.894 & 0.863 & 0.879 & - \\
\hline & Group B & $530.8 \pm 24.4$ & $531.8 \pm 20.3$ & $533.2 \pm 17.4$ & $523.0 \pm 17.7$ & $528.7 \pm 19.8$ & $530.4 \pm 21.2$ & 0.561 \\
\hline & $p$-value ${ }^{\ddagger}$ & - & 0.823 & 0.734 & 0.584 & 0.624 & 0.913 & - \\
\hline & $p$-value ${ }^{\S}$ & 0.658 & 0.547 & 0.411 & 0.208 & 0.232 & 0.253 & - \\
\hline \multirow{5}{*}{$\begin{array}{l}\text { Pupil } \\
\text { size } \\
(\mathrm{mm})\end{array}$} & Group A & $4.16 \pm 0.30$ & $4.05 \pm 0.50$ & $4.06 \pm 1.12$ & $3.99 \pm 0.51$ & $3.91 \pm 0.42$ & $4.07 \pm 0.38$ & 0.557 \\
\hline & $p$-value ${ }^{\dagger}$ & - & 0.812 & 0.845 & 0.451 & 0.419 & 0.877 & - \\
\hline & Group B & $4.08 \pm 0.56$ & $4.09 \pm 0.45$ & $4.11 \pm 0.97$ & $4.16 \pm 0.44$ & $4.18 \pm 0.64$ & $4.16 \pm 0.33$ & 0.641 \\
\hline & $p$-value & - & 0.863 & 0.831 & 0.792 & 0.755 & 0.792 & - \\
\hline & $p$-value $e^{\S}$ & 0.574 & 0.758 & 0.715 & 0.459 & 0.422 & 0.612 & - \\
\hline
\end{tabular}

Values are presented as mean \pm standard deviation; Group A: combined cataract operation (phacoemulsification with intraocular lens implantation) and vitrectomy; Group B: cataract operation (phacoemulsification with intraocular lens implantation).

$\mathrm{ACD}=$ anterior chamber depth; $\mathrm{ACV}=$ anterior chamber volume; $\mathrm{ACA}=$ anterior chamber angle; CCT $=$ central corneal thickness. ${ }^{*}$ Friedman test to analyze changes after Nd:YAG laser capsulotomy; ${ }^{\dagger}$ Paired $t$-test compared with pretreatment data (group A); ${ }^{\star}$ Paired $t$-test compared with pretreatment data (group B); ${ }^{\S}$ Mann-Whitney $U$-test for comparisons between groups A and B.

Visual acuity, intraocular pressure, and spherical equivalent

Visual acuity improved significantly from 1 hour to 3 months after capsulotomy in both groups, but the differences between these two groups were not significant (Table 3). Capsulotomy did not significantly alter the IOP in either group, and there were no significant differences between groups. The spherical equivalent (SE) in group A was -0.29 \pm 0.98 diopters (D) before capsulotomy, $-0.32 \pm 0.57 \mathrm{D}$ at 1 day after capsulotomy, and $-0.32 \pm 0.22 \mathrm{D}$ at 3 months after capsulotomy, showing a non-significant tendency toward myopic shift $(p=0.072)$. In group $\mathrm{B}$, the $\mathrm{SE}$ was $-0.33 \pm$ $0.46 \mathrm{D}$ before capsulotomy, $-0.40 \pm 0.89 \mathrm{D}$ at 1 hour after capsulotomy, $-0.48 \pm 0.29 \mathrm{D}$ at 1 day after capsulotomy, and $-0.49 \pm 0.25 \mathrm{D}$ at 3 months after capsulotomy; this tendency toward myopic shift was not quite significant ( $p=$ $0.055)$.

\section{Discussion}

Current cataract surgery requires precise determination of the IOL position to minimize postoperative refractive 
Table 3. Changes in VA, IOP, and SE after Nd:YAG laser capsulotomy

\begin{tabular}{|c|c|c|c|c|c|c|c|c|}
\hline & & Pre-capsulotomy & 1 Hour & 1 Day & 1 Week & 1 Month & 3 Months & $p$-value ${ }^{*}$ \\
\hline \multirow{5}{*}{$\begin{array}{l}\text { VA } \\
\text { (logMAR) }\end{array}$} & Group A & $0.46 \pm 0.33$ & $0.16 \pm 0.12$ & $0.15 \pm 0.32$ & $0.13 \pm 0.09$ & $0.14 \pm 0.03$ & $0.14 \pm 0.12$ & $<0.001$ \\
\hline & $p$-value ${ }^{\dagger}$ & - & $<0.001$ & $<0.001$ & $<0.001$ & $<0.001$ & $<0.001$ & - \\
\hline & Group B & $0.48 \pm 0.32$ & $0.15 \pm 0.22$ & $0.12 \pm 0.14$ & $0.12 \pm 0.08$ & $0.11 \pm 0.06$ & $0.11 \pm 0.11$ & $<0.001$ \\
\hline & $p$-value & - & $<0.001$ & $<0.001$ & $<0.001$ & $<0.001$ & $<0.001$ & - \\
\hline & $p$-value ${ }^{\S}$ & 0.832 & 0.792 & 0.472 & 0.237 & 0.566 & 0.672 & - \\
\hline \multirow{5}{*}{$\begin{array}{l}\text { IOP } \\
(\mathrm{mmHg})\end{array}$} & Group A & $15.3 \pm 2.1$ & $15.1 \pm 2.3$ & $14.8 \pm 1.5$ & $15.1 \pm 2.5$ & $15.2 \pm 1.7$ & $15.3 \pm 0.7$ & 0.421 \\
\hline & $p$-value ${ }^{\dagger}$ & - & 0.842 & 0.753 & 0.823 & 0.872 & 0.891 & - \\
\hline & Group B & $15.9 \pm 1.5$ & $16.1 \pm 1.3$ & $15.7 \pm 1.2$ & $15.8 \pm 1.2$ & $15.4 \pm 1.9$ & $15.0 \pm 1.6$ & 0.875 \\
\hline & $p$-value & - & 0.862 & 0.873 & 0.831 & 0.773 & 0.745 & - \\
\hline & $p$-value ${ }^{\S}$ & 0.921 & 0.563 & 0.617 & 0.824 & 0.963 & 0.588 & - \\
\hline \multirow{5}{*}{$\begin{array}{l}\mathrm{SE} \\
\text { (diopter) }\end{array}$} & Group A & $-0.29 \pm 0.98$ & $-0.29 \pm 1.02$ & $-0.32 \pm 0.57$ & $-0.32 \pm 0.32$ & $-0.33 \pm 0.13$ & $-0.32 \pm 0.22$ & 0.072 \\
\hline & $p$-value ${ }^{\dagger}$ & - & 0.894 & 0.822 & 0.822 & 0.831 & 0.822 & - \\
\hline & Group B & $-0.33 \pm 0.46$ & $-0.40 \pm 0.89$ & $-0.48 \pm 0.29$ & $-0.48 \pm 0.59$ & $-0.49 \pm 0.38$ & $-0.49 \pm 0.25$ & 0.055 \\
\hline & $p$-value ${ }^{\ddagger}$ & - & 0.232 & 0.344 & 0.344 & 0.351 & 0.351 & - \\
\hline & $p$-value ${ }^{\S}$ & 0.794 & 0.372 & 0.166 & 0.132 & 0.083 & 0.078 & - \\
\hline
\end{tabular}

Values are presented as mean \pm standard deviation; Group A: combined cataract operation (phacoemulsification with intraocular lens implantation) and vitrectomy; Group B: cataract operation (phacoemulsification with intraocular lens implantation).

$\mathrm{VA}=$ visual acuity; $\mathrm{IOP}=$ intraocular pressure; $\mathrm{SE}=$ spherical equivalent; logMAR = logarithm of the minimum angle of resolution. "Friedman test to compare changes after Nd:YAG laser capsulotomy; ${ }^{\dagger}$ Paired $t$-test compared with pretreatment data (group A); ${ }^{*}$ Paired $t$-test compared with pretreatment data (group B); ${ }^{\S} \mathrm{Mann}$-Whitney $U$-test for comparisons between groups A and B.

error. Accurate IOL positioning is becoming even more important with the increased use of multifocal IOLs. Parameters such as the axial length, ACD, lens thickness, CCT, pupil size, and the presence of vitreous bodies can affect IOL positioning [14-16]. Changes in anterior and posterior chamber parameters, resulting from changes in IOL position after Nd:YAG laser capsulotomy, can cause visual disturbances and alter the refractive index. Thus, it is important to assess changes in anterior and posterior chamber parameters after capsulotomy.

Similar to the findings of the present study, a previous study reported significant reductions in the ACD, as measured with a Pentacam, after Nd:YAG laser capsulotomy in eyes that developed PCO after cataract surgery [12]. In contrast to our findings, one study reported that the ACD after capsulotomy increases in proportion to capsulorhexis size [10], while another found no significant changes in the ACD after Nd:YAG laser capsulotomy for PCO [7,9]. Thus, studies assessing changes in the ACD after Nd:YAG laser capsulotomy for the treatment of PCO after cataract surgery have yielded inconsistent results.

Although several studies have considered the effects of
Nd:YAG laser capsulotomy in eyes that developed PCO after cataract surgery, data are lacking on the changes in the ACD after Nd:YAG laser capsulotomy to treat PCO after combined phacovitrectomy. The present study found that $\mathrm{Nd}$ :YAG laser capsulotomy did not significantly alter the ACD in this group of patients. Removal of the vitreous body may reduce pressure from the posterior to the anterior segment after Nd:YAG laser posterior capsulotomy, resulting in no significant change in the ACD.

Several studies have reported myopic shift after combined phacovitrectomy [17]. The present study found a tendency toward myopic shift after capsulotomy in patients who underwent combined phacovitrectomy, but the change in the SE was not significant. The lack of significant myopic shift might be due to lack of significant change in the $\mathrm{ACD}$, and the tendency toward myopic shift might be due to removal of the vitreous body, which affects the refractive index. Although the refractive indices of the vitreous body and aqueous humor are almost equal in theory, the refractive index of the vitreous body is slightly higher than that of the aqueous humor in vivo [18]. Therefore, replacement of the vitreous body by aqueous humor after vitrecto- 
my may reduce the refractive index, causing refractive changes such as myopic shift $[19,20]$. On the other hand, patients in group B who underwent cataract surgery alone showed a significant decrease in the $\mathrm{ACD}$, while patients in group $\mathrm{B}$ showed a non-significant tendency toward myopic shift ( $p=0.055$ ). These results might be explained by the effect of the remaining vitreous body. Conflicting results have been reported for refractive changes due to Nd:YAG laser capsulotomy after cataract surgery. One study observed a myopic shift in the SE after capsulotomy in patients who underwent cataract surgery alone [12], while two other studies reported no significant differences in the SE [7,9]. Thus, there have been no conclusions about refractive changes after Nd:YAG laser capsulotomy to treat PCO after cataract surgery, and further well-designed studies are needed.

The present study found no significant changes in the IOP after Nd:YAG laser capsulotomy and no significant differences in the IOP between the two groups. Similar results have been found in studies assessing changes in the IOP after capsulotomy in patients with PCO that developed after cataract surgery [7,9,12].

The present study had some limitations, including a retrospective design. A prospective study involving more patients may be required to confirm the results. Although equal laser power was used in most patients, and we tried to make each capsulotomy the same size, there may have been some differences in total laser energy or capsulotomy size between patients. Furthermore, the results of this study need to be analyzed with a greater number of patients because the low power (5.1\% to $36.4 \%$ ) of some of the parameter results may have made it difficult to detect significant differences. Despite these limitations, to our knowledge, the present study is the first to investigate changes in anterior chamber parameters after Nd:YAG laser posterior capsulotomy in patients with $\mathrm{PCO}$ who underwent combined phacovitrectomy. In conclusion, we found that anterior chamber parameters, such as the $\mathrm{ACD}, \mathrm{ACV}$, and $\mathrm{ACA}$, as well as the refractive index, did not change significantly after Nd:YAG laser capsulotomy to treat PCO in patients who underwent combined cataract surgery and pars plana vitrectomy.

By contrast, patients who underwent cataract surgery alone showed significant reductions in the ACD without significant refractive changes. The results of the present study may help determine the power of the intraocular lens in patients who underwent combined surgery or cataract surgery and who will be undergoing Nd:YAG laser capsulotomy.

\section{Conflict of Interest}

No potential conflict of interest relevant to this article was reported.

\section{References}

1. Khambhiphant B, Liumsirijarern C, Saehout P. The effect of Nd:YAG laser treatment of posterior capsule opacification on anterior chamber depth and refraction in pseudophakic eyes. Clin Ophthalmol 2015;9:557-61.

2. MacEwen CJ, Dutton GN. Neodymium-YAG laser in the management of posterior capsular opacification: complications and current trends. Trans Ophthalmol Soc $U K$ 1986;105(Pt 3):337-44.

3. Schaumberg DA, Dana MR, Christen WG, Glynn RJ. A systematic overview of the incidence of posterior capsule opacification. Ophthalmology 1998;105:1213-21.

4. Awasthi N, Guo S, Wagner BJ. Posterior capsular opacification: a problem reduced but not yet eradicated. Arch Ophthalmol 2009;127:555-62.

5. Wormstone IM, Wang L, Liu CS. Posterior capsule opacification. Exp Eye Res 2009;88:257-69.

6. Hu CY, Woung LC, Wang MC. Change in the area of laser posterior capsulotomy: 3 month follow-up. $J$ Cataract Refract Surg 2001;27:537-42.

7. Hu CY, Woung LC, Wang MC, Jian JH. Influence of laser posterior capsulotomy on anterior chamber depth, refraction, and intraocular pressure. J Cataract Refract Surg 2000;26:1183-9.

8. Aslam TM, Devlin H, Dhillon B. Use of Nd:YAG laser capsulotomy. Surv Ophthalmol 2003;48:594-612.

9. Ozkurt YB, Sengor T, Evciman T, Haboglu M. Refraction, intraocular pressure and anterior chamber depth changes after Nd:YAG laser treatment for posterior capsular opacification in pseudophakic eyes. Clin Exp Optom 2009;92:412-5.

10. Findl O, Drexler W, Menapace R, et al. Changes in intraocular lens position after neodymium: YAG capsulotomy. $J$ Cataract Refract Surg 1999;25:659-62.

11. Thornval P, Naeser K. Refraction and anterior chamber depth before and after neodymium: YAG laser treatment 
for posterior capsule opacification in pseudophakic eyes: a prospective study. J Cataract Refract Surg 1995;21:457-60.

12. Oztas Z, Palamar M, Afrashi F, Yagci A. The effects of Nd:YAG laser capsulotomy on anterior segment parameters in patients with posterior capsular opacification. Clin Exp Optom 2015;98:168-71.

13. Eliacik M, Bayramlar H, Erdur SK, et al. Anterior segment optical coherence tomography measurement after neodymium-yttrium-aluminum-garnet laser capsulotomy. Am J Ophthalmol 2014;158:994-8.

14. Olsen T. Calculation of intraocular lens power: a review. Acta Ophthalmol Scand 2007;85:472-85.

15. Park SH, Park KH, Kim JM, Choi CY. Relation between axial length and ocular parameters. Ophthalmologica 2010;224:188-93.
16. Olsen T. Prediction of the effective postoperative (intraocular lens) anterior chamber depth. J Cataract Refract Surg 2006;32:419-24.

17. Hamoudi H, La Cour M. Refractive changes after vitrectomy and phacovitrectomy for macular hole and epiretinal membrane. J Cataract Refract Surg 2013;39:942-7.

18. Sardar DK, Swanland GY, Yow RM, et al. Optical properties of ocular tissues in the near infrared region. Lasers Med Sci 2007;22:46-52.

19. Shioya M, Ogino N, Shinjo U. Change in postoperative refractive error when vitrectomy is added to intraocular lens implantation. J Cataract Refract Surg 1997;23:1217-20.

20. Mehdizadeh M, Nowroozzadeh MH. Postoperative induced myopia in patients with combined vitrectomy and cataract surgery. J Cataract Refract Surg 2009;35:798-9. 